\title{
Opensource
}

\section{Denken Sie an Redefreiheit, nicht an Freibier!}

\author{
Rainer H. Bubenzer, Hamburg
}

NOTFALL \& HAUSARZTMEDIZIN 2004; 30: A 176-A 177

„Denken Sie an Redefreiheit, nicht an Freibier!“ ('Think free speech, not free beer'), so einer der schillerndsten Vertreter der Opensource-Bewegung, Richard Stallman, Gründer der Free Software Foundation (FSF) zu den Grundgedanken hinter Opensource. Also entweder Software, deren Quelltext frei erhältlich ist („quelloffen“) oder Software, die frei zur Verfügung steht („offene Quelle“) (1). Vielen - nicht Opensource-Bewegten - stellt sich die Frage, wie und womit die Entwickler freier Software eigentlich Geld verdienen, wenn nicht mit dem Verkauf(LizenzVergabe) ihrer Produkte.

A uch die klassisch-homöopathisch arbeitenden Ärzte kennen das Prinzip: Sie geben oft die paar Globuli eines individuell gewählten Homöopathikums gleich während der Konsultation an den Patienten. Und zwar ohne dies zu berechnen (schließlich gibt es ja kein Selbstdispensierrecht mehr). Berechnet wird hingegen die - oft extrem aufwändige - Dienstleistung der homöopathischen Anamnese und Arzneimittelfindung. Bezogen auf die EDV ist dies den meisten Praxisinhabern mit eigener Datenverarbeitungsanlage mehr als verständlich: Sie mussten nämlich feststellen, dass aufwändige Investitionen in Hard- und in Software alleine nicht ihr Hauptproblem lösen konnten: Nämlich das teure EDV-System unter den speziellen Bedingungen der jeweiligen Praxis zum Laufen zu bringen und dann an die individuellen Bedürfnisse, zum Beispiel von Arzt und Mitarbeitern, anzupassen.

Natürlich versucht der Verkäufer einer Computeranlage, der Anbieter

\section{Tab. 1 Opensource-Infos}

- GNU Operating System - Free Software Foundation (www.gnu.org/home.html)

- Möller E. GNU/Linux und Open Source: Die Reformation zum Anfassen (6-teilige Serie). Telepolis 12.10.2001 ff (www.heise.de/tp/ deutsch/inhalt/te/9786/1.html) eines Betriebssystems oder der Hersteller einer Arztpraxissoftware zu verschleiern, dass die wahren Kosten die Basisinvestitionen weit übertreffen. Moderne Berechnungsverfahren können dabei helfen, reale Kosten und Nutzen bei der Anschaffung von Software und Hardware abzuschätzen (TCO - Total cost of ownership). Und dabei nicht nur die Anschaffungskosten, sondern alle Aspekte der späteren Nutzung und Wartung der betreffenden Computer-Komponenten zu berücksichtigen. Auch beim Auto sollten die Besitzer schließlich nicht nur den Benzinpreis pro 100 Kilometer bedenken. Wenn also im Folgenden Opensource-Software vorgestellt wird, denken Sie bitte nicht nur an die Ersparnisse beim Kauf der Software, sondern berücksichtigen Sie die Folgekosten, die Installation, Anpassung und Wartung auch bei jeder OpensourceSoftware mit sich bringen.

\section{Was ist Linux?}

Lange vor den meisten bekannten Betriebssystemen wie Windows, Mac OS u. a. gab es schon Unix. Unix ist also eigentlich ein altes Betriebssystem. Dennoch erscheint es technisch recht modern, weil es von Anfang an mit Merkmalen ausgestattet war, die beispielsweise von Microsoft erst sehr viel später (seit Windows NT) in vergleichbarer Form angeboten wurden. So gab es unter Unix von Anfang an echtes Multitasking, eine Trennung der Prozesse voneinander (d. h. hohe Stabilität), klar definierte $\mathrm{Zu}-$ griffsrechte für Dateien (d. h. hohe Sicherheit im Multiuser-Betrieb), ausgereifte Netzwerkfunktionen etc. Allerdings bot Unix vor ein bis zwei Jahrzehnten nur eine spartanische Benutzeroberfläche und stellte hohe Hardware-Anforderungen. Linux (2) ist im Prinzip nichts anderes als eine neue Unix-Variante. Zu seinen Besonderheiten zählen die freie Verfügbarkeit des gesamten Quelltexts, aber auch die breite Hardware-Unterstützung. Die enorme Verbreitung, die Linux in den letzten Jahren gefunden hat, aber auch der Internet-Boom mit dem damit verbundenen großen Bedarf an stabilen und skalierbaren Hochleistungsnetzwerk-Servern, hat der schon totgesagten Unix-Welt neuen Auftrieb gegeben (Tab. 1).

Linux ist jedoch mehr als nur der Kern eines Betriebssystems („Kernel“). Es ist vielmehr das riesige Bündel von Programmen, das mit einer Linux-Distribution typischerweise mitgeliefert wird: Dazu zählen neben unzähligen kleinen Tools und Werkzeugen die Desktop-Systeme KDE und Gnome, verschiedene Office-Pakete, das Zeichenprogramm Gimp, zahllose Programmiersprachen und mindestens ebenso viele Server-Programme (z. B. Webserver). Die zumeist auf $C D$ oder DVD verkauften Distributionen ermöglichen eine rasche und bequeme Installation von Linux. Sie unterscheiden sich jedoch in zahlreichen Eigenschaften:

\section{Umfang, Aktualität}

Die Anzahl, Auswahl und Aktualität der mitgelieferten Programme und Bibliotheken unterscheidet sich von Distribution zu Distribution. 
Manche überbieten sich in der Anzahl der mitgelieferten CDs. Um den Anwendern bei der Installation beziehungsweise bei späteren Updates ein ständiges CD-Wechseln zu ersparen, werden manche Distributionen auch als DVD ausgeliefert.

\section{Installations- und}

\section{Konfigurationswerkzeuge}

Die mitgelieferten Programme zur Installation, Konfiguration und Wartung des Systems helfen dabei, Hunderte von Konfigurationsdateien des Systems auf einfache Weise richtig einzustellen. Funktionierende Installations- und Konfigurationstools stellen eine enorme Zeitersparnis dar.

\section{Konfiguration des Desktop (KDE, Gnome)}

Viele Distributionen lassen dem Anwender die Wahl zwischen KDE, Gnome und eventuell auch verschiedenen Window-Managern. Es gibt aber auch Unterschiede in der Detailkonfiguration von KDE oder Gnome, die das Aussehen, die Menüanordnung etc. betreffen.

\section{Hardware-Erkennung und-Konfiguration}

Linux kommt mit den meisten PC-Hardware-Komponenten zurecht. Angenehm ist natürlich, wenn die Distribution Ihre Hardware automatisch erkennt und damit umgehen kann.

\section{Paketsystem}

Die Verwaltung von Linux-Anwendungsprogrammen erfolgt durch Pakete. Das Paketsystem hat Einfluss darauf, wie einfach die Nachinstallation zusätzlicher Programme beziehungsweise das Update vorhandener Programme ist. Zurzeit sind drei zueinander inkompatible Paketsysteme üblich:

- RPM (unter anderem bei Mandrake, Red Hat, SUSE, TurboLinux),

- DEB (Debian) oder

- TGZ(Slackware).

\section{Wartung, Sicherheits-Updates}

Linux ist ein sich dynamisch veränderndes System. Oft gibt es nach der Fertigstellung einer Distribution noch wichtige Neuerungen; immer wieder mal werden Sicherheitsmängel entdeckt. Eine gute Distribution zeichnet sich dadurch aus, dass es ein halb- oder vollautomatisches Update-System gibt, um SicherheitsUpdates mühelos $\mathrm{zu}$ installieren (Tab. 2, Tab. 3).

\section{DER kostenlose Microsoft Office-Ersatz}

Wenn Sie bislang MS Office verwenden, gibt es eine vollwertige Opensource-Alternative: OpenOffice (www.openoffice.org, ist auf fast allen Linux-Distributions-CDs bereits enthalten - sparen Sie sich den Download), ein Paket mit nahezu allen Funktionen, die auch das teure Pendant enthält. Die kommerzielle Variante von OpenOffice, nämlich StarOffice (wwws.sun.com/software/star/staroffice, ca. 75 Euro), enthält noch einige zusätzliche Eigenschaften (z. B. weitere Importfilter oder die Datenbank Adabas), die jedoch nicht für alle Anwender notwendig sind. Einige vergleichende Anmerkungen finden Sie in der Computerzeitschrift Chip: „Sun StarOffice 7 - Microsoft Office 2003: Der Vergleich hinkt" (www.chip.de/artikel/ c_artikelunterseite_11078711.html)

(Tab. 4).

\section{Tab. 2 Wichtige Linux-Distributionen (Auswahl)}

$\begin{array}{ll}\text { S.u.S.E Linux } & \text { www.suse.de } \\ \text { Knoppix GNU/Linux } & \text { www.knopper.net } \\ \text { Debian GNU/Linux } & \text { www.debian.org } \\ \text { RedHat Linux } & \text { www.redhat.com } \\ \text { Caldera Open Linux } & \text { www.caldera.com } \\ \text { Deutsche Linux Distribution } & \text { www.delix.de }\end{array}$

\section{Weiterführende Linux-Infos}

1. Hetze S, Hohndel D, Kirch O, Müller M. Das Linux Anwenderhandbuch und Leitfaden für die Systemverwaltung, www.linux-ag.de/linux/LHB/index.html

2. www.linux.de

3. www.linux.org

4. The Linux Documentation Project - en.tldp.org

\section{Literatur}

1. NN: Open Source. Wikipedia - Die freie Enzyklopädie, 2004. de.wikipedia.org/wiki/ Opensource (Wikipedia ist eine „Opensource"-Wissendatenbank)

2. Kofler M. Linux - Installation, Konfiguration, Anwendung. 7. Auflage. AddisonWesley, München. 2004. ISBN 3827321581 (inkl. DVD mit SUSE Linux v9.x; www. kofler.cc/linux.html).

\section{Anschrift des Verfassers \\ Rainer H. Bubenzer \\ Medizin- und Wissenschaftsjournalist \\ (DJV, KdM) \\ multi MED vision \\ Borselstraße 9, 22765 Hamburg \\ Fax: 0 40/41912877 \\ E-Mail: Rainer@Bubenzer.com}

\section{Tab. 3 Abrechnungsprogramme unter Linux (Beispiele)}

\begin{tabular}{lll} 
Name & Hersteller & im Internet \\
\hline DAVID classic & Data Vital GmbH \& Co. KG & www.data-vital.de \\
\hline Adamed plus Linux & DOCexpert Computer GmbH & www.docexpert.de \\
\hline APRIS & APRIS Praxiscomputer GmbH & www.apris.de \\
\hline $\begin{array}{l}\text { APW-Arztpraxis } \\
\text { Wiegand (DOS) }\end{array}$ & $\begin{array}{l}\text { APW-Wiegand-Medizinische Software, } \\
\text { Entwicklung und Vertrieb GmbH }\end{array}$ & www.apw-wiegand.de \\
\hline TurboMed Classic (DOS) & TurboMed EDV GmbH & www.turbomed.de \\
\hline
\end{tabular}

Abrechnungsprogramme, die unter DOS laufen, können mit entsprechendem Emulator auch auf einem Linux-System arbeiten. Eine Anleitung, wie dies mit Knoppix, DOSemu und FreeDOS bei dem Arztpraxis Wiegand zu realisieren ist, findet sich hier: resmedicinae.sourceforge.net/manuals/apw_linux/knxdos.html. Entsprechend sollte dies auch mit anderen DOS-Programmen funktionieren.

Auch Windows-Abrechnungsprogamme sind „irgendwie“ unter Linux lauffähig. Das Vorgehen: Zuerst Linux und dann den Windows-Emulator VMWare Workstation für Linux (www.vmware.com) installieren. Anschließend können Windows und beliebige Windowsprogramme installiert werden. Tipp: Diskutieren Sie nie mit einem Linux-Anhänger über die Sinnhaftigkeit dieses Vorgehens (betriebswirtschaftlich ist es nämlich sinnlos)!

\section{Tab. 4 Freie Software und Medizin}

- OpenMedPortal - Freie Software für die Medizin (www.openmed.org/html/index.php)

- Res Medicinae - Information in Medicine (resmedicinae.sourceforge.net)

- Diskussionsliste zur Medizinischen Informatik (lists.sourceforge.net/lists/listinfo/resmedicinae-deutsch)

- Journal of Free and Open Source Medical Computing (JOSMC) (www.josmc.org)

- Linux-Forum Deutsches Ärzteblatt (www.aerzteblatt.de/v4/foren/beitrag.asp?id=35762)

- Freie Praxissoftware GNUmed (www.gnumed.de) 